\title{
Almost all graphs with $2.522 n$ edges are not 3-colorable
}

\author{
Dimitris Achlioptas * \\ Michael Molloy ${ }^{\dagger}$ \\ optas@cs.toronto.edu \\ molloy@cs.toronto.edu \\ Department of Computer Science \\ University of Toronto \\ Toronto, Ontario M5S 3G4, Canada.
}

\begin{abstract}
We prove that for $c \geq 2.522$ a random graph with $n$ vertices and $m=c n$ edges is not 3 -colorable with probability $1-o(1)$. Similar bounds for non- $k$-colorability are given for $k>3$.
\end{abstract}

1991 Mathematics Subject Classification: Primary 05C80; Secondary 05C15.

\section{Introduction}

Let $N(n, m, A)$ denote the number of graphs with vertices $\{1, \ldots, n\}, m=m(n)$ edges and some property $A$. The term "almost all" in the title has the meaning introduced by Erdös and Rényi [5]:

$$
\lim _{n \rightarrow \infty} \frac{N(n, m, A)}{\left(\begin{array}{c}
n \\
2 \\
m
\end{array}\right)}=1
$$

Equivalently, one can consider a random graph $G=G(V, E)$ where $|V|=n$ and $E$ is a uniformly random $m$-subset of all $\left(\begin{array}{l}n \\ 2\end{array}\right)$ possible edges on $V$, i.e. the $G(n, m)$ model of random graphs. If $n$ is an index running over probability spaces, we will say that a sequence of events $\mathcal{E}_{n}$ occurs with high probability (w.h.p.) if $\lim _{n \rightarrow \infty} \operatorname{Pr}\left[\mathcal{E}_{n}\right]=1$. In particular, we will say that " $G(n, m(n))$ has property $A$ w.h.p." if $m(n)$ is such that (1) holds for $A$.

In their seminal paper introducing random graphs [5], Erdős and Rényi pointed out that a number of interesting properties exhibit a sharp threshold behavior on $G(n, m)$ : for each such property $A$, there exists a critical number of edges $m_{A}(n)$ such that for $m$ around

${ }^{*}$ Researh supported in part by an NSERC PGS Scholarship. Current address: Microsoft Research, One Microsoft Way, Redmond WA 98052, U.S.A. Email: optas@microsoft.com

${ }^{\dagger}$ Researh supported in part by an NSERC grant. 
$m(n)$ the probability of $G(n, m)$ having $A$ changes rapidly from near 0 to near 1 . Such properties include having a multicyclic component, having a perfect matching, connectivity, Hamiltonicity and others.

A central property in this context is the $k$-colorability of $G(n, c n)$ where $k$ is a fixed integer. For $k=2$, this is very well-understood as bipartiteness is equivalent to containing no odd cycles. In particular, the probability of non-2-colorability is bounded away from 0 for any $c>0$ and keeps increasing gradually with $c$, reaching $1-o(1)$ during the emergence of the giant component at $c=1 / 2$. For $k>2$, though, our understanding of $k$-colorability is not nearly as good; moreover, the situation is conjectured to be quite different. In particular, see $[5,3]$, Erdős asked: for each $k>2$, is there a constant $c_{k}$ such that for any $\epsilon>0$,

$$
G\left(n,\left(c_{k}-\epsilon\right) n\right) \text { is w.h.p. } k \text {-colorable and } G\left(n,\left(c_{k}+\epsilon\right) n\right) \text { is w.h.p. not } k \text {-colorable ? }
$$

Recently, Friedgut [6] made great progress in our understanding of threshold phenomena in random graphs by establishing necessary and sufficient conditions for a property to have a sharp threshold. Using the main theorem of [6], Friedgut and the first author [1] showed that for $k>2$, there exists a function $t_{k}(n)$ such that $(2)$ holds upon replacing $c_{k}$ with $t_{k}(n)$, i.e. that indeed $k$-colorability has a sharp threshold. While it is widely believed that $\lim _{n \rightarrow \infty} t_{k}(n)$ exists, confirming this conjecture and determining the limit $c_{k}$, even for $k=3$, seems very challenging.

Perhaps the main tool in attacking the question of $k$-colorability for small values of $k>2$ has been the elementary fact that if a graph has no subgraph with minimum degree at least $k$, then it is $k$-colorable. In particular, first Łuczak [11] proved that w.h.p. $G(n, c n)$ remains 3-colorable after the emergence of the giant component by showing that for $c \leq 0.50005$, w.h.p. $G(n, c n)$ has no subgraph of minimum degree 3. Shortly afterwards, Chvátal [4] improved this greatly by showing that $G(n, c n)$ w.h.p. has no subgraph with minimum degree 3 for $c \leq 1.44$ and Reed and the second author [13] improved the bound even further to $c \leq 1.67$. Finally, Pittel, Spencer and Wormald [16], proved that, in fact, for all $k>2$ there exists $\gamma_{k}$ such that for $c<\gamma_{k}, G(n, c n)$ w.h.p. has no subgraph with minimum degree at least $k$, while for $c>\gamma_{k}$ it has such a subgraph w.h.p. Moreover, they determined $\gamma_{k}$ exactly for all $k$, in particular yielding $c_{3} \geq \gamma_{3}=1.675 \ldots$ Following that, and in aswering a question of Bollobás [3], the second author [14] proved $c_{k}>\gamma_{k}$ for all $k \geq 4$ and conjectured $c_{3} \neq \gamma_{3}$ as well. This conjecture was verified recently by the authors [2] after analyzing the performance of a greedy "list-coloring" heuristic on $G(n, c n)$. That argument yielded $c_{3}>1.923$, which is the best known lower bound for $c_{3}$.

In this paper, after briefly reviewing the known upper bounds for $c_{k}$, we show how a technique of Kirousis et al. [8], developed for random $k$-SAT, can be used to yield an improved upper bound for $c_{k}$ for small values of $k$. For example, we obtain

\section{Theorem 1}

$$
c_{3}<2.522 \text {. }
$$




\section{The first moment method}

Grimmett and McDiarmid [7] gave the first lower bound on the chromatic number of random graphs by determining $\alpha_{k}$ such that $G\left(n, m=\alpha_{k} n^{2}\right)$ w.h.p. has no independent set of size $n / k$, and thus $\chi(G)>k$ (here $k \rightarrow \infty$ ). Moreover, they conjectured that the lower bound derived by this argument is tight, and as evidence for this they showed that the expected number of $k$-colorings of $G\left(n, \alpha n^{2}\right)$ tends to infinity for $\alpha<\alpha_{k}$. Devroye (see [4]) later observed that when $k$ is fixed, letting the expected number of $k$-colorings go to 0 as $n \rightarrow \infty$ yields much better lower bounds for the chromatic number than letting the number of (suitably large) independent sets go to 0 as $n \rightarrow \infty$.

Our proof can be viewed as a refinement of Devroye's argument which we will reproduce below to introduce some ideas and notation. Before doing so, let us recall that in the $G(n, m)$ model the edge set is a random $m$-subset of the set of all $\left(\begin{array}{l}n \\ 2\end{array}\right)$ possible edges. Equivalently, we can say that the edges of the graph are selected from the set of all possible edges one-by-one, uniformly, independently and without replacement. For the calculations in this paper it will be convenient to consider a slight modification of the $G(n, m)$ model in that the selection is done with replacement, i.e. multiple edges are allowed. We will denote this model by $G^{r}(n, m)$. Intuitively, it is clear that for any monotone increasing property $A$ and any value of $m$, the probability of $A$ holding in $G(n, m)$ is no smaller than it is in $G^{r}(n, m)$ since "additional occurrences of an edge do not help". Formally, this is Theorem 5 in [9] and, for our purposes, it will imply that if for a given $m(n), G^{r}(n, m(n))$ is w.h.p. non- $k$-colorable then so is $G(n, m(n))^{*}$.

We will distinguish between a proper $k$-coloring of a graph and one in which some adjacent vertices might have the same color by referring to them as a " $k$-coloring" and a " $k$-partition", respectively. In fact, it will be helpful to think of a $k$-coloring of a graph $G(V, E)$ as a $k$-partition of $V$ such that every $e \in E$ has its endpoints in distinct blocks of the partition, so that each block is an independent set.

Let $P=V_{1}, \ldots, V_{k}$ be an arbitrary (ordered) $k$-partition of $V$ and let $C_{P}$ denote the event that $P$ is a $k$-coloring of $G$. For $C_{P}$ to hold, every edge of the random graph has to connect vertices from two different blocks. Introducing

$$
T(P)=\sum_{i<j}\left|V_{i}\right| \cdot\left|V_{j}\right|
$$

the total number of pairs of vertices belonging to different blocks, we have

$$
\operatorname{Pr}\left[C_{P}\right]=\left(\frac{T(P)}{\left(\begin{array}{l}
n \\
2
\end{array}\right)}\right)^{m} .
$$

*In fact, it turns out that since the expected number of multiple edges in $G^{r}(n, c n)$ is $O(1)$ the converse holds as well, i.e. if $G(n, c n)$ is w.h.p. non- $k$-colorable then so is $G^{r}(n, c n)$. Thus, by switching to the $G^{r}(n, m)$ model we are not giving anything away with respect to bounding $c_{k}$. 
Now, using the fact $\sum_{i}\left|V_{i}\right|=n$ and the Cauchy-Schwartz inequality, respectively, we bound

$$
\begin{aligned}
T(P) & =\frac{n^{2}}{2}-\frac{1}{2} \sum_{i}\left|V_{i}\right|^{2} \\
& \leq \frac{n^{2}}{2}-\frac{1}{2} \cdot \frac{n^{2}}{k} \\
& =\frac{k-1}{2 k} n^{2} .
\end{aligned}
$$

Thus, (4) yields

$$
\operatorname{Pr}\left[C_{P}\right] \leq\left(\frac{k-1}{k}\right)^{m}\left(\frac{n}{n-1}\right)^{m} .
$$

Since the number of $k$-partitions of $V$ is $k^{n}$, (5) implies that the expected number of $k$-colorings of $G$, for $m=c n$, is of order

$$
\left[k\left(\frac{k-1}{k}\right)^{c}\right]^{n} .
$$

Hence, if $c>\frac{\ln k}{\ln k-\ln (k-1)}$ the expected number of $k$-colorings of $G^{r}(n, c n)$ tends to 0 as $n \rightarrow \infty$ implying that $G^{r}(n, c n)$ is w.h.p. non- $k$-colorable. For $k=3$, this argument yields $c_{3}<2.71$ and in general $c_{k}<k \ln k$.

It is worth noting that this simple argument is asymptotically tight: the upper bound on $\chi(G(n, m))$ given by Euczak [10] implies that for any $\epsilon>0$ and all $k \geq k_{0}(\epsilon), c_{k}>(1-\epsilon) k \ln k$. On the other hand, the following two observations can be used to show that for $k>2$ this argument is not exact: (a) if a $k$-colorable graph has $s_{i}$ vertices of degree $i$ then it has at least $\prod_{i=0}^{k-1}(k-i)^{s_{i}}$ distinct $k$-colorings and (b) with extremely high probability, for every fixed $i$, $G(n, c n)$ has $\Omega(n)$ vertices of degree $i$. If $X$ is the number of $k$-colorings of $G(n, c n)$, using (a),(b), one can show that there are values of $c$ such that for some $a>b>1$ : (i) $\mathbf{E}[X] \approx b^{n}$ and (ii) w.h.p. if $X>0$ then $X>a^{n}$. Hence, for such $c, \operatorname{Pr}[X>0] \leq(a / b)^{n}+o(1)=o(1)$, while $\mathbf{E}[X]$ is exponentially large. Thus, it is not the case that $G(n, c n)$ is w.h.p. $k$-colorable for exactly those values of $c$ for which its expected number of $k$-colorings is large.

Indeed, Reed and the second author [13] proved that this "naive" first moment argument is quite a bit off the mark for $k=3$. To that end, they first extended the argument to uniformly random pseudographs on a given degree sequence (for a definition see also [15]). In particular, they proved that such a pseudograph with $\rho n$ edges is w.h.p. non- $k$-colorable if $\rho>\frac{\ln k}{\ln k-\ln (k-1)}$. Then, in order to improve over the naive bound, they considered the random pseudograph resulting by repeatedly (20 times) removing all vertices of degree less than 3 from $G(n, m=c n)$. They proved that this pseudograph (i) is uniformly random with respect to its degree sequence and (ii) if $c \geq c_{0}=2.571 \ldots$, then w.h.p. it has at least $\rho n$ edges where $\rho>\frac{\ln 3}{\ln 3-\ln 2}$. Hence, w.h.p. $G\left(n, m=c_{0} n\right)$ contains a non-3-colorable subgraph, implying $c_{3}<2.572$.

Inspired by the work of Kirousis et al. [8], we will take a less direct but more fruitful approach towards accounting for the wastefulness of the first moment method. Instead of 
focusing on the low degree vertices explicitly, we will prove the following: if $P$ is a $k$-coloring of $G \in G(n, c n)$ and we randomly pick a vertex $v$, then with probability $\phi=\phi(k, c)>0$ we can assign a different color to $v$ and still have a $k$-coloring of $G$. This suggests that when $k$-colorings exist, they tend to appear in large "clusters" of similar colorings. The approach of Kirousis et al. [8], when translated to coloring, suggests that instead of counting all the $k$-colorings of a random graph (as the first moment does) we should only count a few "representative" ones. Following this idea we will consider as representatives those $k$-colorings satisfying a certain "local maximality" condition and determine their expected number in $G^{r}(n, c n)$. Letting that expectation go to 0 as $n \rightarrow \infty$ will yield $c_{3}<2.522$.

\section{A refinement of the first moment method}

Recall that for a $k$-partition $P=V_{1}, V_{2}, \ldots, V_{k}$ of $V, C_{P}$ denotes the event that $P$ is a $k$-coloring of $G$. Let us say that a vertex $v \in V_{i}$ is unmovable in $P$ if for every $j>i$ the partition resulting by moving $v$ to $V_{j}$ is not a $k$-coloring of $G$. We will say that $P$ is a rigid $k$-coloring of $G$ if $C_{P}$ holds and every vertex is unmovable in $P$. We will denote this event by $R_{P}$. Note now that if we consider the $k$-partitions of $V$ as strings of length $n$ over $\{1, \ldots, k\}$ then, clearly, the lexicographically last $k$-coloring of $G$ (if any $k$-coloring exists) is rigid by definition. Hence, $G$ has a rigid $k$-coloring iff it is $k$-colorable, implying that the probability that $G^{r}(n, c n)$ is $k$-colorable is bounded by the expected number of rigid $k$-colorings of $G^{r}(n, c n)$. With this in mind, we take $m=c n$ and seek $c=c(k)$ for which this last expectation tends to 0 as $n \rightarrow \infty$.

Remark: Note that requiring $k$-colorings to be rigid, immediately eliminates all the redundant counting caused by vertices of degree $k-1$ or less; only the $k$-colorings which assign every such vertex the greatest possible color get counted.

\subsection{Probability Calculations}

For every $k$-partition $P=V_{1}, V_{2}, \ldots, V_{k}$ of $V$ we let

$$
\alpha_{i}=\alpha_{i}(P)=\frac{\left|V_{i}\right|}{n}
$$

Also, recalling (3), we let

$$
\tau=\tau(P)=\frac{T(P)}{n^{2}} .
$$

It is well-known that for any $c>0$, the largest independent set of $G(n, c n)$ w.h.p. contains only a constant fraction of all vertices. Thus, the probability that $G(n, c n)$ has a $k$-coloring where only one color class contains $\Omega(n)$ vertices is $o(1)$. Hence, in the following we only consider partitions $P$ in which at least two blocks have $\Omega(n)$ vertices (and bound the expected number of rigid $k$-colorings among such partitions).

We will first bound $\operatorname{Pr}\left[R_{P}\right]$. For this, using (5), it suffices to bound $\operatorname{Pr}\left[R_{P} \mid C_{P}\right]$. 
For a given $k$-coloring $P$, any $i$, any vertex $v \in V_{i}$, and any $j>i$ we let $\mathcal{E}(v, j)$ denote the event " $v$ cannot be moved to $V_{j}$ ". Thus,

$$
\operatorname{Pr}\left[R_{P} \mid C_{P}\right]=\operatorname{Pr}\left[\bigcap_{\substack{i<j \\ v \in V_{i}}} \mathcal{E}(v, j) \mid C_{P}\right] .
$$

Letting $E(v, j)=\left\{\{v, w\}: w \in V_{j}\right\}$, we see that $\mathcal{E}(v, j)$ occurs iff at least one member of $E(v, j)$ is an edge of $G$. Note that since we have conditioned on $C_{P}$, only two-element sets $\{v, w\}$ enumerated by $T(P)$ can appear in the graph. Thus, since the edges of $G$ were chosen uniformly, independently and with replacement,

$$
\begin{aligned}
\operatorname{Pr}\left[\mathcal{E}(v, j) \mid C_{P}\right] & =1-\left(1-\frac{\left|V_{j}\right|}{T(P)}\right)^{m} \\
& =1-e^{-\alpha_{j} c / \tau}+O\left(n^{-1}\right),
\end{aligned}
$$

where the passage from (8) to (9) relies on the fact that $P$ has more than one blocks with $\Omega(n)$ vertices and, thus, $T(P)=\Omega\left(n^{2}\right)$. (This is our only use of the fact that there are more than one blocks with $\Omega(n)$ vertices.)

To bound $\operatorname{Pr}\left[R_{P} \mid C_{P}\right]$ using (7),(9) we first observe that the sets $E(v, j)$ induce a partition of the set of two-element sets $\{v, w\}$ enumerated by $T(P)$, since each $\{v, w\}$ where $v \in V_{i}$, $w \in V_{j}$ and $i<j$ belongs to exactly one such set, namely $E(v, j)$. Since the total number of edges is fixed and each event $\mathcal{E}(v, j)$ "consumes" at least one edge of $E$, it is intuitively clear that the events $\mathcal{E}(v, j)$ should be negatively correlated. To prove this assertion, we view the formation of $E$ (conditional on $C_{P}$ ) as an allocation scheme with $m$ distinguishable balls, $T(P)$ boxes, and a partition of the set of boxes into disjoint subsets $E(v, j),\left(v \in V_{i}, i<j\right)$. Thus, the occurrence of $\mathcal{E}(v, j)$ simply means that the total occupancy of boxes from $E(v, j)$ is at least one. Now, the negative correlation of the events $\mathcal{E}(v, j)$ follows from a classical result of McDiarmid [12]. As a result we get

$$
\operatorname{Pr}\left[R_{P} \mid C_{P}\right] \leq \prod_{\substack{i<j \\ v \in V_{i}}} \operatorname{Pr}[\mathcal{E}(v, j)],
$$

and, thus, using (7),(9) and (10) we get

$$
\begin{aligned}
\operatorname{Pr}\left[R_{P} \mid C_{P}\right] & \leq \prod_{1 \leq i<j \leq k}\left(1-e^{-\alpha_{j} c / \tau}+O\left(n^{-1}\right)\right)^{\alpha_{i} n} \\
& =\left(\prod_{2 \leq j \leq k}\left(1-e^{-\alpha_{j} c / \tau}\right)^{\sum_{i<j} \alpha_{i}}\right)^{n} \times O(1) .
\end{aligned}
$$

Having bounded $\operatorname{Pr}\left[R_{P} \mid C_{P}\right]$, we bound the expected number of rigid $k$-colorings, $\mathrm{E}[R(G)]$, as follows. For $k$-partitions $P_{1}=V_{1}^{1}, \ldots, V_{k}^{1}$ and $P_{2}=V_{1}^{2}, \ldots, V_{k}^{2}$, we say that 
THe EleCtronic Journal of Combinatorics 6 (1999), \#R29

$P_{1}$ is isomorphic to $P_{2}$ if $\left|V_{i}^{1}\right|=\left|V_{i}^{2}\right|$, for all $i$. Clearly, if $P_{1}, P_{2}$ are isomorphic then $\operatorname{Pr}\left[R_{P_{1}}\right]=\operatorname{Pr}\left[R_{P_{2}}\right]$. Let $\mathcal{P}$ be any maximal set of non-isomorphic $k$-partitions of $V$. Then

$$
\begin{aligned}
\mathbf{E}[R(G)] & =\sum_{P} \operatorname{Pr}\left[R_{P}\right] \\
& =\sum_{P \in \mathcal{P}}\left(\begin{array}{c}
n \\
\alpha_{1} n, \ldots, \alpha_{k} n
\end{array}\right) \operatorname{Pr}\left[R_{P}\right] \\
& \leq \max _{P \in \mathcal{P}}\left[\left(\begin{array}{c}
n \\
\alpha_{1} n, \ldots, \alpha_{k} n
\end{array}\right) \operatorname{Pr}\left[R_{P}\right]\right] n^{k-1},
\end{aligned}
$$

as there are at most $n^{k-1}$ (ordered) partitions of $n$ into $k$ integers. Moreover, if $n>0$ and all $\alpha_{i} n$ are integers it is well-known that

$$
\left(\begin{array}{c}
n \\
\alpha_{1} n, \ldots, \alpha_{k} n
\end{array}\right)<\left(\frac{1}{\alpha_{1}^{\alpha_{1}} \cdots \alpha_{k}^{\alpha_{k}}}\right)^{n}, \text { where } 0^{0} \equiv 1
$$

Thus, combining (4),(6) and (11)-(13) we have

$$
\mathbf{E}[R(G)] \leq\left(\max _{P \in \mathcal{P}} f(P)\right)^{n} \times O\left(n^{k-1}\right)
$$

where

$$
f(P)=\frac{\left(2 \sum_{i<j} \alpha_{i} \alpha_{j}\right)^{c}}{\alpha_{1}^{\alpha_{1}} \cdots \alpha_{k}^{\alpha_{k}}} \prod_{2 \leq j \leq k}\left(1-e^{-\alpha_{j} c / \tau}\right)^{\sum_{i<j} \alpha_{i}} .
$$

Letting $Q=\{q / n: q \in\{0, \ldots, n\}\}$, it is clear that maximizing $f$ over $P \in \mathcal{P}$ amounts to maximizing the right-hand side of (15) over $Q^{k}$ subject to $\sum_{i} \alpha_{i}=1$. Naturally, we still get an upper bound on $\mathbf{E}[R(G)]$ if we relax each such $\alpha_{i}$ to an arbitrary real number in $[0,1]$ and maximize the extended function, $g$, over $D=[0,1]^{k}$ subject to $\sum_{i} \alpha_{i}=1$. If for some $c^{*}=c^{*}(k)$ the resulting maximum of $g$ is strictly less than 1, then (14) implies that $\mathbf{E}[R(G)] \rightarrow 0$ as $n \rightarrow \infty$ and, thus, that $G^{r}\left(n, c^{*} n\right)$ is w.h.p. non- $k$-colorable.

It is straightforward to verify that $g$ is continuous, differentiable and its gradient is bounded on $D$. As a result, $g$ can be maximized numerically with arbitrarily good, guaranteed precision (we used Maple [18] and the code in [17]). For example, for $k=3$ we have

$$
g\left(\alpha_{1}, \alpha_{2}, \alpha_{3}\right)=\frac{\left(2 \tau_{3}\right)^{c}\left(1-e^{-\alpha_{2} c / \tau_{3}}\right)^{\alpha_{1}}\left(1-e^{-\alpha_{3} c / \tau_{3}}\right)^{\alpha_{1}+\alpha_{2}}}{\alpha_{1}^{\alpha_{1}} \alpha_{2}^{\alpha_{2}} \alpha_{3}^{\alpha_{3}}}
$$

where $\tau_{3}=\alpha_{1} \alpha_{2}+\alpha_{1} \alpha_{3}+\alpha_{2} \alpha_{3}$. For $c^{*}=2.5217, g$ is maximized around $\alpha_{1}=0.30746$, $\alpha_{2}=0.33527, \alpha_{3}=0.35727$ and at that vicinity it is strictly less than 0.9999744 . Thus, $G^{r}\left(n, m=c^{*} n\right)$ is w.h.p. non- $k$-colorable, implying $c_{3}<2.522$.

Similarly, we get the following new bounds for $c_{k}$ for $3 \leq k \leq 7$. (The choice of 7 is rather arbitrary, as the numerical computations remain manageable for substantially larger $k$.) 


\begin{tabular}{|l|c|c|c|c|c|}
\hline$k$ & 3 & 4 & 5 & 6 & 7 \\
\hline First moment bound & 2.710 & 4.819 & 7.213 & 9.828 & 12.714 \\
\hline New bound & 2.522 & 4.587 & 6.948 & 9.539 & 12.316 \\
\hline
\end{tabular}

The above table gives an idea of how our improvement over the first moment bound scales with $k$. Recalling that the first moment bound is asymptotically tight, we see that already for $k=7$ the improvement has dropped to less than $3 \%$ from $7 \%$ for $k=3$.

It seems clear that one could improve the upper bound on $c_{k}$ somewhat further by imposing a stricter local maximality condition. For example, one could consider conditions that involve "moving" two vertices at a time. Unfortunately, the lack of "independence" between the outcomes of different moves in that setting seems to complicate matters greatly.

\section{Acknowledgements}

We would like to thank the authors of [8] for providing us with an early draft of their paper and an anonymous referee for many valuable comments.

\section{References}

[1] D. Achlioptas, E. Friedgut, A sharp threshold for $k$-colorability, Random Structures $\&$ Algorithms, 14 (1999), 63-70.

[2] D. Achlioptas, M. Molloy, The analysis of a list-coloring algorithm on a random graph, 38th Annual Symposium on Foundations of Computer Science, Miami, FL (1997), 204-212.

[3] N. Alon, J.H. Spencer, The Probabilistic Method, with an Appendix of open problems by P. Erdős, J. Wiley \& Sons, New York, 1992.

[4] V. Chvátal, Almost all graphs with $1.44 n$ edges are 3-colorable, Random Structures $\&$ Algorithms, 2 (1991), 11-28.

[5] P. Erdős, A. Rényi, On the evolution of random graphs, Publication of the Mathematical Institute of the Hungarian Academy of Sciences, 5 (1960), 17-61.

[6] E. Friedgut, Necessary and sufficient conditions for sharp thresholds of graph properties, and the $k$-SAT problem, Journal of the American Mathematical Society, 12 (1999), $1017-1054$.

[7] G.R. Grimmett, C.J.H. McDiarmid, On colouring random graphs, Mathematical Proceedings of the Cambridge Philosophical Society, 77 (1975), 313-324.

[8] L. M. Kirousis, E. Kranakis, D. Krizanc, and Y. Stamatiou, Approximating the unsatisfiability threshold of random formulas, Random Structures \& Algorithms, 12 (1998), $253-269$. 
[9] L. M. Kirousis, Y. Stamatiou, An inequality for reducible increasing properties of random words, Technical Report, Computer Technology Institute, Greece, (1997), 1-3.

[10] T. Łuczak, The chromatic number of random graphs, Combinatorica, 11 (1991), 45-54.

[11] T. Euczak, Size and connectivity of the $k$-core of a random graph, Discrete Mathematics, 91 (1991), 61-68.

[12] C.J.H. McDiarmid, On a correlation inequality of Farr, Combinatorics, Probability and Computing, 1 (1992), 157-160.

[13] M. Molloy, The chromatic number of sparse random graphs, M. Math Thesis, University of Waterloo, (1992).

[14] M. Molloy, A gap between the appearances of a $k$-core and a $(k+1)$-chromatic graph, Random Structures 85 Algorithms, 8 (1996), 159-160.

[15] M. Molloy, B. Reed, A critical point for random graphs with a given degree sequence, Random Structures 85 Algorithms, 6 (1995), 161-179.

[16] B. Pittel, J.H. Spencer, and N.C. Wormald, Sudden emergence of a giant $k$-core in a random graph, Journal of Combinatorial Theory Series B, 67 (1996), 111-151.

[17] W.H. Press, S. Teukolsky, W.T. Vetterling, and B.P. Flannery, Numerical recipes in C, Cambridge University Press, Cambridge, 1992.

[18] D. Redfern, The Maple Handbook: Maple V Release 3, Springer, New York, 1994. 